\title{
A NOTE ON THE LOWER DERIVATE OF A SET FUNCTION AND SEMIHEREDITARY SYSTEMS OF SETS
}

\author{
W. F. PFEFFER
}

1. Introduction. Every Perron-like definition of an integral, that is to say, a definition which uses major and minor functions, is based essentially on the following theorem:

"An additive set function is nonnegative whenever its lower derivate is nonnegative."

In its classical setting for an additive function of compact intervals, the theorem is well-known and rather easy to prove (see $[8$, p. 190, Theorem 3.1]). However, once we proceed from the family of compact in tervals to more general systems of sets, the situation becomes far more complicated. The basic trouble lies in the fact that for a general system of sets we define additivity by using disjoint rather than nonoverlapping sets (compare the definitions in $[3$, p. 11] and in $[8$, p. 61]). Some difficulties occur even when we deal with an additive function of all Lebesgue measurable subsets of the unit interval. To illustrate the problem we shall start with this special case. The general situation will be treated in $\$ 4$. There we shall formulate and prove our main results in a purely topological manner using the notion of a semihereditary system of sets. In $\$ 5$ we shall prove the theorem concerning the lower derivate by an easy application of the results of $\S 4$.

2. Special case. By $\mathbb{R}$ we shall denote the family of all Lebesgue measurable subsets of the unit interval $[0,1]$. For $A \in \mathbb{R},|A|$ and $d(A)$ will denote the Lebesgue measure and the diameter of $A$, respectively. Let $F$ be a real-valued function on $\Omega$ and let $x \in[0,1]$. We shall call the number

$$
D_{*}(F, x)=\lim _{t \rightarrow 0+}\left[\inf _{A \in \mathbb{R}(x, t)} \frac{F(A)}{|A|}\right],{ }^{1}
$$

where $\mathfrak{R}(x, t)=\{A \in \mathbb{R}: x \in A$ and $d(A)<t\}$, the lower derivate of $F$ at $x$. The function $F$ is said to be additive whenever $F(B \cup C)=F(B)$ $+F(C)$ for all disjoint sets $B, C \in \mathbb{R}$.

Received by the editors August 24, 1966.

1 We let $a / 0=+\infty$ for $\mathrm{a}>0$ and $a / 0=-\infty$ for $a<0$; the symbols $a /( \pm \infty)$ and $0 / 0$ are not defined. 
2.1. Proposition. Let $F$ be an additive function on $\mathbb{R}$ and let $D_{*}(F, x) \geqq 0$ for all $x \in[0,1]$. Then $F(A) \geqq 0$ for every closed set $A \in \mathbb{R}$.

Proof. Given $\epsilon>0$, we let $G(B)=F(B)+\epsilon|B|$ for all $B \in \mathbb{R}$. Then $D_{*}(G, x) \geqq D_{*}(F, x)+\epsilon>0$ for all $x \in[0,1]$. Since $G$ is additive and finite, $G(\varnothing)=0$. Suppose there is a closed set $A \in \mathbb{R}$ such that $G(A)<0$. We denote by $M$ the set of all $x \in[-1,1]$ for which $G(A \cap[-1, x])$ $<0$. Obviously $1 \in M$ and $M$ is a subset of the interval $[0,1]$. Let $x_{0}=\inf M$ and let $\left\{x_{n}\right\},\left\{y_{n}\right\},-1<x_{n}<x_{0}, y_{n} \in M, n=1,2, \cdots$, be two sequences such that $\lim x_{n}=\lim y_{n}=x_{0}$. Because $G$ is additive, $G\left(A \cap\left[-1, y_{n}\right]\right)<0$ and $G\left(A \cap\left[-1, x_{n}\right]\right) \geqq 0$, we have $G\left(A \cap\left(x_{n}, y_{n}\right]\right)$ $<0$. Hence, $A \cap\left(x_{n}, y_{n}\right] \neq \varnothing$ and we can choose $z_{n} \in A \cap\left(x_{n}, y_{n}\right]$, $n=1,2, \cdots$. Since $A$ is closed and $\lim z_{n}=x_{0}$, it follows that $x_{0} \in A$. Thus $D_{*}\left(G, x_{0}\right) \leqq 0$ which is a contradiction. The proposition now follows from the arbitrariness of $\epsilon$.

Proposition 2.1 cannot be sharpened as the following example shows. For $A \in \mathbb{R}$ let

$$
F(A)=\chi_{A}(0)-\operatorname{LIM}[|A \cap[0,1 / n]| /|[0,1 / n]|],
$$

where $\chi_{A}$ is the characteristic function of $A$ and LIM is a generalized Banach limit (see [2, p. 73, Example 22]). It is not too hard to check that $F$ is an additive function on $\&$ and that $D_{*}(F, x)=0$ for every $x \in[0,1]$. Nevertheless, $F((0,1])=-1$.

3. Preliminaries. In this section we state definitions and notation which are needed subsequently.

3.1. Definition. Let $\alpha$ be a system of sets. An extended realvalued function $F$ defined on $\alpha$ is said to be superadditive if and only if

$$
F(A \cup B) \geqq F(A)+F(B)
$$

for all $A, B \in \alpha$, with $A \cup B \in \alpha$ and $A \cap B=\varnothing$, for which $F(A)+F(B)$ has meaning. Replacing the inequality sign $\geqq$ by the equality sign $=$, we obtain the definition of an additive function.

3.2. Definition. Let $\alpha$ be a system of sets. The system of sets $\sigma$ is said to be semihereditary with respect to $\alpha$ if and only if for every $A \in \sigma$ and every $B \in \alpha$ either $A \cap B \in \sigma$ or $A-B \in \sigma$.

3.3. Proposition. Let $\alpha$ be a system of sets which is closed with respect to the formation of set differences. ${ }^{2}$ If $F$ is a superadditive function on $\alpha$, then $\alpha^{F}=\{A \in \alpha: F(A)<0\}$ is semihereditary with respect to $\alpha$. On the other hand, if $\sigma \subset \alpha$ is semihereditary with respect to $\alpha$, then there is a superadditive function $F$ on $\alpha$ such that $\alpha^{F}=\sigma$.

\footnotetext{
${ }^{2}$ In particular, this is true when $\alpha$ is a ring.
} 
Proof. Let $A \in \alpha^{F}$ and $B \in \alpha$. Because $F(A-B)+F(A \cap B)$ $\leqq F(A)<0^{3}$ whenever the sum has meaning, it follows that either $A-B$ or $A \cap B$ belongs to $\alpha^{F}$. In order to prove the second part of the proposition it suffices to let $F(A)=-1$ for $A \in \sigma$ and $F(A)=0$ for $A \in \alpha-\sigma$.

Proposition 3.3 shows the close relationship between Definitions 3.1 and 3.2. Later we shall see that the semihereditariness of the system $\alpha^{F}$ is quite sufficient to prove the theorem about the lower derivate.

3.4. Notation. For any system of sets $\alpha$ and any set $A$, we let $\alpha_{A}=\{B \in \alpha: \varnothing \neq B \subset A\}$.

If $\sigma$ is semihereditary with respect to $\alpha$, so is $\sigma_{A}$ for every set $A$. If $\beta \subset \alpha$ and $\sigma$ is semihereditary with respect to $\alpha$, it is also semihereditary with respect to $\beta$.

4. Main theorems. From now on we shall assume that $P$ is a locally compact Hausdorff topological space and that $P^{-}=P \cup(\infty)$ is a onepoint compactification of $P$ (see [6, pp. 117, 150]). If $A \subset P^{-}$, then $A^{-}$and $A^{0}$ denote the closure and the in terior of $A$ in $P^{-}$, respectively. For $x \in P^{-}, \mathfrak{B}_{x}$ will be a local base at $x$ in $P^{-}$(see $[6$, p. 50$\left.]\right)$. We shall also assume that we have already chosen a system $\alpha \subset \exp P{ }^{4}$ If we now say $\sigma \subset \exp P$ is semihereditary, we mean that $\sigma$ is semihereditary with respect to $\alpha$. The following assumptions about the system $\alpha$ will be used:

(i) For every $x \in P, \mathfrak{B}_{x} \subset \alpha$.

(ii) If $A \in \alpha$ and $C \subset A^{0}$ is compact, then there is a $B \in \alpha$ such that $C \subset B^{0} \subset B^{-} \subset A^{0}$.

4.1. Proposition. Let the system $\alpha$ be closed with respect to the formation of set differences. Then $\alpha$ satisfies (ii) whenever it satisfies (i).

Proof. Let $A \in \alpha$ and let $C \subset A^{0}$ be compact. From (i) and the regularity of $P^{-}$, it follows that every $x \in C$ has a neighborhood $U_{x} \in \alpha$ such that $U_{x}^{-} \subset A^{0}$. Because $C$ is compact, there are $x_{i} \in C$, $i=1,2, \cdots, n$, for which $C \subset \bigcup_{i=1}^{n} U_{x_{i}}^{0}$. Letting $B=\bigcup_{i=1}^{n} U_{x_{i}}$, we obtain $C \subset B^{0} \subset B^{-} \subset A^{0}$ and

$$
B=A-(A-B)=A-\bigcap_{i=1}^{n}\left(A-U_{x_{i}}\right) .
$$

${ }^{3}$ A system of sets which is closed with respect to the formation of set differences is also closed with respect to the formation of set intersections; for $A \cap B=A-$ $(A-B)$.

${ }^{4} \exp P$ is the collection of all subsets of $P$. 
The proposition follows (see footnote 3 ).

4.2. Theorem. Let $\sigma \subset \exp P$ be a semihereditary system and let $\varnothing \neq A \in \sigma$. If $\alpha$ satisfies condition (i), then there is an $x \in A^{-}$such that every neighborhood $U$ of $x$ contains a set $B \in \sigma_{A}$.

Proof. Suppose the theorem is not true. Then every $x \in A^{-}$has a neighborhood $U_{x} \in \mathfrak{W}_{x}$ which does not contain any set $B \in \sigma_{A}$. Because $A^{-}$is compact, there are points $x_{i} \in A^{-}, i=1,2, \cdots, n$, such that $A \subset \bigcup_{i=1}^{n} U_{x_{i}}$ and no proper subfamily of $\left\{U_{x_{i}}\right\}_{i=1}^{n}$ covers $A$. According to (i), we may assume that $U_{x_{1}}, \cdots, U_{x_{n-1}}$ belong to $\alpha$. From the choice of $U_{x_{1}}$ and the semihereditariness of $\sigma_{A}$, it follows that $A-U_{x_{1}} \in \sigma_{A}$. Suppose we have already proved that $A-\bigcup_{i=1}^{n-2} U_{x_{i}}$ $\in \sigma_{A}$. Again from the choice of $U_{x_{n-1}}$ and the semihereditariness of $\sigma_{A}$ we can conclude that

$$
A-\bigcup_{i=1}^{n-1} U_{x_{i}}=\left(A-\bigcup_{i=1}^{n-2} U_{x_{i}}\right)-U_{x_{n-1}} \in \sigma_{A} .
$$

This contradicts the choice of $U_{x_{n}} ;$ for $A-\cup_{i=1}^{n-1} U_{x_{i}} \subset U_{x_{n}}$.

We note that neither the local compactness nor the Hausdorff separability of the space $P$ was used in the proof of Theorem 4.2. This theorem holds for an arbitrary topological space $P$.

4.3. Theorem. Let $\sigma \subset \exp P$ be a semihereditary system and let $\varnothing \neq A \in \sigma$. If $\alpha$ satisfies conditions (i) and (ii), then there is an $x \in A^{-}$ such that every neighborhood $U$ of $x$ contains a set $B \in \sigma_{A}$ for which $x \in B^{-}$. If, in addition, $A$ is compact, then there is an $x \in A$ such that every neighborhood $U$ of $x$ contains a set $B \in \sigma_{A}$ for which $x \in B$.

Proof. By $\Gamma$ we shall denote the system of all nonempty compact sets $C \subset P^{-}$with the following property: if $G \subset P^{-}$is an open set containing $C$, then there is a set $Q \in \sigma_{A}$ such that $Q \subset G$ and $C \subset Q^{-}$. The system $\Gamma$ is not empty because $A^{-} \in \Gamma$. Let $\Gamma_{0} \subset \Gamma$ be a nonempty chain (see [6, p. 32]) and let $C_{0}=\bigcap_{C \in \Gamma_{0}} C$. Obviously $C_{0}$ is nonempty and compact. Given an open set $G \subset P^{-}$containing $C_{0}$, we have $\bigcap_{C \in \Gamma_{0}}(C-G)=C_{0}-G=\varnothing$. Because $\left\{C-G: C \in \Gamma_{0}\right\}$ is a chain of compact sets, there is $C \in \Gamma_{0}$ such that $C \subset G$. Now it readily follows that $C_{0} \in \Gamma$. According to $[6$, p. 33, Theorem 25(b)], there is a set $D \in \Gamma$ which is minimal in $\Gamma$.

If $D$ contains two different points $y$ and $z$, we may assume that $y \neq \infty$. Since $(y) \notin \Gamma$, there is an open set $G \subset P^{-}$such that $y \in G$ and no subset of $G$ which contains $y$ in its closure belongs to $\sigma_{A}$. From (i) it follows that $y$ has a neighborhood $U \in \alpha$ such that $U \subset G-(z)$. So $D-U^{0}$ is a nonempty compact proper subset of $D$. Let $H \subset P^{-}$ 
be an open set containing $D-U^{0}$. Without loss of generality we may assume that $y \notin H$. Because $D-H$ is a compact subset of $U^{0}$, from (ii) it follows that there is $V \in \alpha$ such that $D-H \subset V^{0} \subset V^{-} \subset U^{0}$. Hence $H \cup V^{0}$ is an open set containing $D$. Because $D \in \Gamma$, there is $Q \in \sigma_{A}$ such that $Q \subset H \cup V^{0}$ and $D \subset Q^{-}$. Since $y \in Q^{-}$and $y \in V^{0}$, $y \in(Q \cap V)^{-}$. We also have $Q \cap V \subset G$. Thus, according to the choice of $G, Q \cap V \notin \sigma_{A}$. From this fact and the semihereditariness of $\sigma_{A}$, it follows that $Q-V \in \sigma_{A}$. Because $Q-V \subset H$ and $D-U^{0} \subset Q^{-}-V^{-}$ $C(Q-V)^{-}$, we conclude that $D-U^{0} \in \Gamma$. This contradicts the minimality of $D$ in $\Gamma$.

We can conclude that $D=(x)$ is a singleton and the first part of the theorem follows from the definition of the system $\Gamma$.

Suppose that $A$ is compact. By $\Lambda$ we shall denote the system of all nonempty compact sets $C \subset P$ with the following property: if $G \subset P$ is an open set containing $C$, then there is a set $Q \in \sigma_{A}$ such that $C \subset Q \subset G$. Now we can repeat verbatim the proof of the first part of the theorem with $\Gamma$ replaced by $\Lambda$. The proof of Theorem 4.3 is completed.

Let us notice that a filter $\Phi$ in $P$ is an ultrafilter if and only if it is semihereditary with respect to $\alpha=\exp P$ (see [1, p. 38, Proposition 5]). From this and $[1$, Chapter $1, \S 10.1]$, follows the essential importance of the compactness of $P^{-}$. Theorems 4.2 and 4.3 generally cannot be proved in the space $P$ alone. It would be interesting to replace condition (ii) by some other condition or, if possible, to omit it entirely. For example, by a slight modification of the given proof, it can be shown that the second part of Theorem 4.2 also holds if we replace conditions (i) and (ii) by the following one:

Every $x \in P$ has an open local base $\mathfrak{B}_{x} \subset \alpha$.

5. Application. Throughout this section we shall assume that the system $\alpha$ is closed with respect to the formation of set differences and satisfies condition (i). Thus, according to $4.1, \alpha$ also satisfies condition (ii). For $x \in P^{-}$and $U \in \mathfrak{W}_{x}$ we denote

$$
\begin{gathered}
\alpha_{1}(x, U)=\alpha_{U}, \quad \alpha_{2}(x, U)=\left\{A \in \alpha_{U}: x \in A^{-}\right\}, \\
\alpha_{3}(x, U)=\left\{A \in \alpha_{U}: x \in A\right\} .
\end{gathered}
$$

5.1. Definition. Let $F$ and $G$ be extended real-valued functions on $\alpha$ and let $x \in P^{-}$. For $i=1,2,3$, we shall call the number

$$
D_{*}^{i}(F, G, x)=\sup _{U \in \mathbb{B}_{x}}\left[\inf _{A \in \alpha_{\imath}(x, U)} \frac{F(A)}{G(A)}\right]
$$

(see footnote 1) the $i$ th lower derivate of $F$ with respect to $G$ at $x$. 
If $P^{-}$is a metric space, Definition 5.1 is a special case of the definition given in $[3$, p. 246$]$ or $[4,2.14]$. In particular, $D_{*}^{3}(F, G, x)$ is a special case of the lower derivate with respect to a natural blanket (see $[5,2.11])$.

5.2. THEOREM. Let $F$ and $G$ be superadditive functions on $\alpha, 0 \leqq G$ $<+\infty$, and let $A \in \alpha$. If $D_{*}^{3}(F, G, x) \geqq 0$ for all $x \in A^{-}$, then $F(B) \geqq 0$ for every compact set $B \in \alpha_{A}$. If $D_{*}^{2}(F, G, x) \geqq 0$ or $D_{*}^{1}(F, G, x) \geqq 0$ for all $x \in A^{-}$, then $F(B) \geqq 0$ for every $B \in \alpha_{A}$.

Proof. Suppose $D_{*}^{3}(F, G, x) \geqq 0$ for all $x \in A^{-}$. Given $\epsilon>0$, we let $H=F+\epsilon G$. It is rather easy to see that

$$
D_{*}^{3}(H, G, x) \geqq D_{*}^{3}(F, G, x)+\epsilon>0
$$

for all $x \in A^{-}$. Thus, according to 3.3 and $4.3, H(B) \geqq 0$ for every compact set $B \in \alpha_{A}$. The first part of Theorem 5.2 now follows from the arbitrariness of $\epsilon$. The proof of the second part of this theorem is similar.

Theorem 5.2 will be used for the definition of a Perron integral in [7].

\section{REFERENCES}

1. N. Bourbaki, Topologie générale, Hermann, Paris, 1951.

2. N. Dunford and G. T. Schwartz, Linear operators, Interscience, New York, 1958.

3. H. Hahn and A. Rosenthal, Set functions, Univ. of New Mexico Press, Albuquerque, 1948.

4. C. A. Hayes, Differentiation with respect to $\phi$-pseudo-strong blankets and related problems, Proc. Amer. Math. Soc. 3 (1952), 283-296.

5. C. A. Hayes and A. P. Morse, Convexial blankets, Proc. Amer. Math. Soc. 6 (1950), 719-730.

6. J. L. Kelley, General topology, Van Nostrand, New York, 1955.

7. W. F. Pfeffer, An integral in topological spaces, (to appear). ${ }^{5}$

8. S. Saks, Theory of the integral, Hafner, New York, 1937.

George Washington University and

University of CALIFornia, Davis

${ }^{5}$ For the abstract see Notices Amer. Math. Soc. 12 (1965), 625-37, p. 555. 